\title{
Cerebellar liponeurocytoma: A case report and review of the literature
}

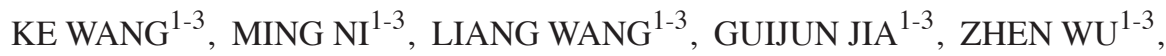 \\ LIWEI ZHANG ${ }^{1-3}$ and JUNTING ZHANG ${ }^{1-3}$ \\ ${ }^{1}$ Skull Base and Brainstem Tumor Division, Department of Neurosurgery, Beijing Tiantan Hospital; \\ ${ }^{2}$ Beijing Institute for Brain Disorders, Brain Tumor Center, Capital Medical University; \\ ${ }^{3}$ China National Clinical Research Center for Neurological Diseases (NCRC-ND), Beijing 100050, P.R. China
}

Received October 25, 2014; Accepted August 17, 2015

DOI: $10.3892 / 01.2015 .3986$

\begin{abstract}
Cerebellar liponeurocytoma is rare, and the clinical characteristics and treatment strategy remain unclear. In the present study, a case of cerebellar liponeurocytoma was retrospectively reported and a literature review was performed. A 45-year-old female presented due to occipital headaches, exhibiting a hoarse voice and a broad-based gait. Pre-operative magnetic resonance images revealed a lesion occupying the right hemisphere of the cerebellum and the inferior vermis, compressing the medulla oblongata from the right side, and extending through the foramen magnum to the C2 level. A total resection was performed, and pathological analysis of the lesion showed positivity for synaptophysin, S-100 and neuronal nuclear antigen, partial positivity for Olig-2, and negativity for glial fibrillary acidic protein and epithelial membrane antigen. In addition, the Ki-67 index was low $(<5 \%)$. Thus, a diagnosis of cerebellar liponeurocytoma was determined. Total resection was successful and the patient was followed up closely. A review of the literature showed that cerebellar liponeurocytoma is mainly located in the cerebellum, with rare extra-cerebellar cases. Certain studies have suggested that the tumor may be located supratentorially and subtentorially, and should be renamed as solely liponeurocytoma. Total resection of the tumor contributes to an improved prognosis, while a subtotal resection and high Ki-67 index lead to recurrence. The tumor is similar to a tumor of low malignancy, with long-term recurrence. Radiation is recommended when there is residual tumor, recurrence or when the $\mathrm{Ki}-67$ is high.
\end{abstract}

Correspondence to: Dr Junting Zhang, Skull Base and Brainstem Tumor Division, Department of Neurosurgery, Beijing Tiantan Hospital, Capital Medical University, 6 Tiantan Xili, Dongcheng, Beijing 100050, P.R. China

E-mail: zhangjunting2003@aliyun.com

Key words: cerebellar liponeurocytoma, liponeurocytoma, recurrence, radiation

\section{Introduction}

Cerebellar liponeurocytoma is a rare tumor of the central never system. Since it was first reported in 1978 (1), several names had been applied, such as lipomatous medulloblastoma, medullocytoma, neurolipocy toma, lipomatous glioneurocytoma and lipidized mature neuroectodermal tumor of the cerebellum (1). In 2000, The World Health Organization (WHO) classified this rare tumor as a distinctive entity, prior to characterizing it as a grade II tumor in 2007 (2). Cerebellar liponeurocytoma is most commonly found in adults, without gender predominance (2-4). Patel et al (4) revealed that 42 cases of cerebellar liponeurocytoma were reported in the English literature between 1978 and 2009, with a male to female ratio of 22:20. Clinical symptoms include the symptoms of the cerebellar dysfunction, such as gait disturbance and uncoordinated movements, which are slowly progressive and generally not detected in the early stages of the disease. Vomiting and progressive visual symptoms begin to present, as a result of increased cranial pressure in the later stages of the disease (5). Preclinical diagnosis is difficult as to date, no typical imaging features have been identified, however, Aker et al (3) reported that the lesion exhibited heterogeneous enhancement on T1-weighted images (3). Pathologically, the tumor is characterized by the focal accumulation of adiposities in an otherwise typical small cell tumor, similar to central neurocytoma, with cerebella and supratentorial locations (2-3). The current preferred treatment strategy is radical surgery, and adjuvant radiotherapy is recommended in cases of residual tumor. As the tumor has potentially long-term malignant characteristics, close follow-up is recommended (2-4). However, the clinical characteristics and optimal treatment strategy of the cerebellar liponeurocytoma remain unclear. The present study reports a case located in the foramen magnum region, and also provides a review of the literature.

\section{Case report}

A 45-year-old female was referred to the Department of Neurosurgery of Beijing Tiantan Hospital (Capital Medical University, Beijing, China) in September 2011 complaining 

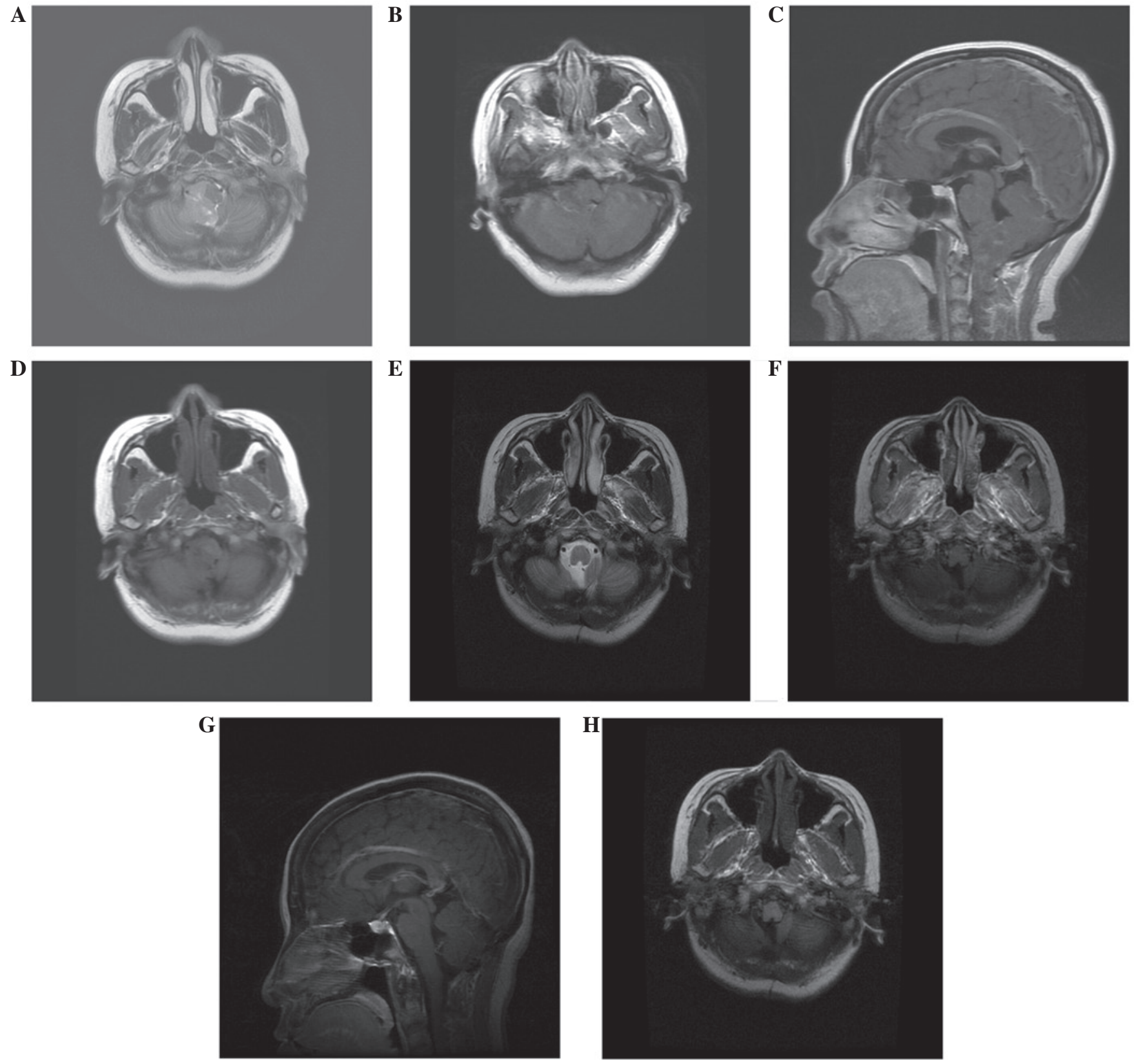

Figure 1. Magnetic resonance imaging (MRI) revealing (A-D) a lesion occupying the right hemisphere of the cerebellum and the inferior vermis, and compressing the medulla oblongata from the right side, with an exophytic component extending through the foramen magnum to the C2 level. (A) On T2WI, the lesion was moderately hyperintense compared with the cortex, the lesion was well-marginated without apparent edema, and no signs of obstructive hydrocephalus were found. (B) Axial and (C) saggital enhanced T1-weighted imaging (WI) revealed moderate heterogeneous enhancement. (D) A sparse hypointense signal was detected on T1WI. (E-H) At 3 years post-surgery, the postoperative (E) T2WI, (F) axial enhanced MRI scan (G) saggital enhanced MRI scan and (H) T1WI showed that the lesion had been completely removed, and there were no signs of recurrence.

of occipital headaches that had persisted for $\sim 18$ months. Paresthesia of the left hand was exhibited prior to slow progression to the other limb, which was associated with debilitation and breathlessness for $\sim 6$ months. The patient had a history of hypertension for $\sim 3$ years, which was controlled with a dietary regimen, exercise therapy and blood pressure monitoring. Upon clinical examination, the patient presented with a hoarse voice, occasional deglutition, diminished pinprick sensations in the right arm and leg, and acroaesthesia of the left side. Muscle strength was normal, however, a broad-based gait was noted. Magnetic resonance imaging (MRI) revealed an isointense lesion with a sparse hypointense signal on T1-weighted imaging (WI) and slightly heterogeneous enhancement on enhanced T1WI. On T2WI, the lesion was slightly hyperintense compared with the cortex (Fig. 1A-D). The lesion was $\sim 4.5 \times 2.5 \times 2.0 \mathrm{~cm}$ in size, occupying the right hemisphere of the cerebellum and the inferior vermis, compressing the medulla oblongata from the right side, and extending through the foramen magnum to the $\mathrm{C} 2$ level. The lesion was well-marginated, as shown by the T2WI, without apparent edema, and no signs of obstructive hydrocephalus were found.

The patient underwent a total resection of the tumor through the midline suboccipital approach, which extended downward 


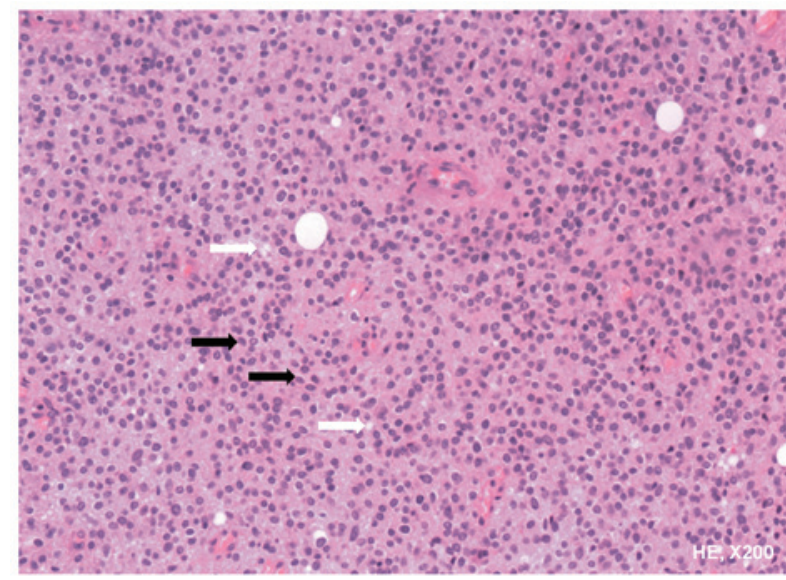

Figure 2. Pathology. Hematoxylin and eosin (HE) staining, revealing monotonous small round cells (black arrow) with moderately eosinophilic cytoplasm. The white arrow shows the sparse lipomatous cells (magnification, $\mathrm{x} 200$ ).

to the $\mathrm{C} 2$ level. During the surgery, the gross appearance of the tumor was noted to be soft and moderately vascularized, with a yellowish color. The tumor was derived from the right cupula region of the fourth ventricle, well demarcated from the surrounding parenchyma, without close adherence to the parenchyma. Microscopic examination showed monotonous small round cells with slightly eosinophilic cytoplasm. The lipomatous cells were localized focally (Fig. 2). Immunohistochemistry was positive for synaptophysin, S-100 and neuronal nuclear antigen, partially positive for Olig-2, and negative for glial fibrillary acidic protein and epithelial membrane antigen. The Ki-67 index was low $(<5 \%)$. The post-operative period was uneventful, with notable improvement of the main symptoms; however, the hoarse voice remained unchanged. Following the total resection of the tumor, radiation therapy was not recommended, and at the 6-month follow-up, there was no evidence of tumor recurrence. In the latest follow-up at 3 years post-surgery, the patient's hoarse voice was significantly recovered and no signs of tumor recurrence were found (Fig. 1E-H).

\section{Discussion}

Little is known with regard to cerebellar liponeurocytoma due to its rarity. As its name implies, cerebellar liponeurocytoma often occurs in the cerebellum, occupying the cerebellar hemisphere, with repletion of the fourth ventricle, causing the clinical symptoms of cranial hypertension and cerebellar symptoms $(2,3)$. The mean age at diagnosis is $\sim 49$ years, and the majority of patients are $>30$ years (4). According to the study by Patel et al, in which 42 cases of liponeurocytoma from the literature were reviewed in 2009, there is no gender predominance (4). The WHO classified cerebellar liponeurocytoma as a grade II tumor, with a tendency to recur in the follow-up period (2).

There has been controversy with regard to the location of cerebellar liponeurocytoma, partly due to its rarity. At first, this tumor appeared to be specifically located to the cerebellum. However, there have been 11 cases reported in an extracerebellar location $(5,6)$. Kuchelmeister et al reported a case located in the lateral ventricle, and confirmed that the liponeurocytoma was not an exclusively cerebellar neoplasm (7). The most notable points with regard to liponeurocytomas of the supratentorial intraventricular region is that there is an absence of calcification and a lack of lipid components when compared with the cerebellar compartment (7). Although the majority of cases in the literature have been located in the cerebellar area, recently, certain studies have suggested that the tumor may be located supratentorially and subtentorially, and should be renamed as solely liponeurocytoma (5-7).

The tumor has a recurrent nature, although this occurs in the long term, with a mean time until recurrence of $\sim 8.5$ years (2). Limaiem et al reported a recurrent cerebellar liponeurocytoma with supratentorial extension, and upon reviewing all 9 recurrent cases presented in the literature, the study suggested that the tumor may recur due to mitoses present in the lesion and $>10 \%$ Ki-67-positive cells (2). However, one confusing element was that, even when treated with total resection, recurrence appeared unavoidable, showing that the cerebellar liponeurocytoma was similar to a malignant lesion (3). To a certain extent, the recurrent tumors were more malignant than the primary lesions, as indicated by the proliferation index and the recurrence period following the second surgery. Anghileri et al reported a case in which a complete resection was performed, and where recurrence was experienced at 8 years and metastasis at 11 years post-surgery, respectively (8). This suggested that the liponeurocytoma was a lesion of uncertain malignant potential.

Châtillon et al analyzed 20 cases, and reported a high recurrence rate in the group treated by gross total resection alone and in the group treated by subtotal resection (9). Although there is no such comparison for the surgery and radiation, there is a trend showing that post-operative radiotherapy benefits the outcome, as suggested by Jackson et al (10). Genc et al reported 2 cases of cerebellar liponeurocytoma with post-surgery $\gamma$-knife therapy, and observed that the tumors diminished in size after a follow-up time of 12 and 53 months, respectively (11). However, certain studies believe that since the recurrence of the tumor occurs in the long term, adjuvant radiation is too aggressive following the initial gross total resection $(2-4,9)$. Considering that there is no evidence to support the use of adjuvant radiation in the prevention of recurrence and due to the side-effects of radiation, the majority of studies in the literature recommended a gross total resection, with a focus on quality of life, and a long-term follow-up plan (7-9). When recurrence was observed, repeat surgery and adjuvant radiation was recommended, as the pathology of the recurrence was always more aggressive than the primary tumor (10-12).

The treatment strategy for the present patient was surgery and close follow-up, since a total resection was achieved, as confirmed intraoperatively and by post-operative MRI, and secondly, since the Ki-67 was low. Radiation therapy was not recommended and close observation was maintained during the follow-up for the subsequent 3 years; no signs of recurrence were found. This treatment strategy was in accordance with those in the literature (7-9), and is recommended for primary tumors with complete resection, in order to avoid aimless radiation and protect the brain.

In summary, the present study reported a case of cerebellar liponeurocytoma that was successfully treated by total resection. A review of the literature showed that cerebellar 
liponeurocytoma is rare and may located supratentorially or subtentorially. The optimal treatment strategy appears to be a total resection and close follow-up, while radiation is recommended when there is residual tumor, recurrence or a high Ki-67. However, due to the rarity of the tumor and limited data available, long-term follow-up is required.

\section{Acknowledgements}

This study was supported, in part, by the Research Special Fund For Public Welfare Industry of Health, China (grant no. 201402008 to Junting Zhang) and the Capital Characteristic Clinic Project, China (grant no. Z131107002213179 to Zhen Wu).

\section{References}

1. Bechtel JT, Patton JM and Takei Y: Mixed mesenchymal and neuroectodermal tumor of the cerebellum. Acta Neuropathol 41: 261-263, 1978.

2. Limaiem F, Bellil S, Chelly I, Bellil K, Mekni A, Jemel H, Haouet S, Zitouna M and Kchir N: Recurrent cerebellar liponeurocytoma with supratentorial extension. Can J Neurol Sci 36: 662-665, 2009.

3. Aker FV, Ozkara S, Eren P, Peker O, Armağan S and Hakan T: Cerebellar liponeurocytoma/lipodized medulloblastoma. J Neurooncol 71: 53-59, 2005
4. Patel N, Fallah A, Provias J and Jha NK: Cerebellar liponeurocytoma. Can J Surg 52: E117-E119, 2009.

5. Pankaj R, Jindal A and Banerjee AK: Liponeurocytoma of lateral ventricle. Neurol India 58: 805-806, 2010.

6. Karabagli P, Sav A and Pamir N: Does "cerebellar liponeurocytoma' always reflect an expected site? An unusual case with a review of the literature. Folia Neuropathol 52: 101-105, 2014.

7. KuchelmeisterK, Nestler U, Siekmann R and Schachenmayr W: Liponeurocytoma of the left lateral ventricle - case report and review of the literature. Clin Neuropathol 25: 86-94, 2006.

8. Anghileri E, Eoli M, Paterra R, Ferroli P, Pollo B, Cuccarini V, Maderna E, Tringali G, Saini M, Salsano E and Finocchiaro G: FABP4 is a candidate marker of cerebellar liponeurocytomas. J Neurooncol 108: 513-519, 2012.

9. Châtillon CE, Guiot MC, Roberge D and Leblanc R: Cerebellar liponeurocytoma with high proliferation index: Treatment options. Can J Neurol Sci 36: 658-661, 2009.

10. Jackson TR, Regine WF, Wilson D and Davis DG: Cerebellar liponeurocytoma. Case report and review of the literature. J Neurosurg 95: 700-703, 2001.

11. Genc A, Bozkurt SU, Karabagli P, Seker A, Bayri Y, Konya D and Kilic T: Gamma knife radiosurgery for cranial neurocytomas. J Neurooncol 105: 647-657, 2011.

12. Chung SB, Suh YL and Lee JI: Cerebellar liponeurocytoma with an unusually aggressive histopathology: Case report and review of the literature. J Korean Neurosurg Soc 52: 250-253, 2012. 